\title{
Content Spending and Network Quality in Mobile Channels: A Hidden Markov Model of User Engagement and Content Consumption
}

\author{
Xia Zhao \\ University of \\ Georgia \\ Athens, GA \\ xia.zhao@uga.edu
}

\author{
Lu Huang \\ Kean University \\ Union, New Jersey \\ lhuang@kean.edu
}

\author{
Lei Wang \\ Penn State University \\ University Park, PA \\ luw21@psu.edu
}

\author{
Elham Yazdani \\ University of Georgia \\ Athens, GA \\ elham.yazdani@uga. \\ edu
}

\author{
Cheng Zhang \\ Fudan University \\ Shanghai, China \\ zhangche@fudan. \\ edu.cn
}

\begin{abstract}
Nowadays, individuals increasingly depend on mobile devices and apps for every aspect of their daily lives. Even though the number of mobile users and the time that they spend on mobile apps have grown tremendously, app providers are still struggling with low user engagement and a high attrition rate. This study examines how users' prior content spending and network delay impact consumers' mobile content consumption decisions and how the impacts vary in different engagement states. We develop a Hidden Markov Model and calibrate it using a large tapstream data set of individual users' reading activities from a mobile app provider. We identify three engagement states and heterogeneous impacts of the financial and operational factors. This study will generate important implications on content pricing and user engagement in mobile channels.
\end{abstract}

\section{Introduction}

Consumers' content consumption has been shifted from the traditional broadcast channels, such as books and magazines, to the Internet channels, and most recently to mobile channels. A Pew Research Center survey shows the number of smartphone users in the U.S. has increased from $35 \%$ in 2011 to $81 \%$ in 2019 [1]. It is also reported that $85 \%$ of U.S. adults got news on a mobile device in 2017, compared to $72 \%$ in 2016 and 54\% in 2013 [2]. An average U.S. adult spent about three hours on his/her smartphones a day, a total of 86 hours a month in 2016 [3]. While individuals embrace the mobile channels for a wide selection of content and the flexibility of consuming the content wherever they are and whenever they want, mobile app providers are still struggling with engaging the users.
Both financial factors such as content spendings and operational factors such as network quality affect user engagement with mobile apps. App providers often embed advertisements to sponsor the content or charge a fee to provide ad-free content. Deloitte's Digital Media Trends survey shows that consumers value the option to view the content without advertisement [4]. A pricing-based revenue model enables app providers to directly monetize the values that mobile content created and potentially leads to a relatively predictable and secured income stream. However, the price-based model poses significant challenges to user engagement and consumption. As users embrace the mobile channels for the availability of a variety of content and the flexibility of consuming the content wherever and whenever they want, they are reluctant to pay for the content. Consumers may stop viewing the paid content or switch to other free content. In addition, consumers, despite consuming a large amount of digital content overall, can only set a short attention span for a piece of content. A Microsoft study reports that the average human attention span has fallen from 12 seconds in 2000 to 8 seconds in 2014, shorter than a goldfish [5]. In mobile computing, users tend to use otherwise wasted time to consume content during commuting via public transportations, waiting in lines, or taking lunch breaks. Even though the consumption is excessive overall, users' engagement with a particular app is often low. Users tend to frequently initiate and end mobile sessions or switch between different contents in a mobile session. Consumers' interests and engagement may decay quickly between sessions. Although the impact of price on product consumption has been documented extensively in the Marketing literature, little is known about how users' prior spending affects the user's engagement states and mobile content consumption.

Network quality is another important factor that impacts individuals' behavior and deserves in-depth 
examination. Individuals have little patience for poor online or mobile experiences. According to Adobe Consumer Content Survey, about 35\% of consumers in the U.S. felt very frustrated about slow page loads, and this number jumps up to $41 \%$ for Millennials [6]. When the loading time was too long, more than half of the consumers stopped viewing content [6]. The relatively small screens of mobile devices may increase users' frustration. Content loading time resulting from network delay would further challenge users' engagement.

To captivate consumers, mobile app providers distribute content in a way that allows consumers to consume the content in a continuous manner, i.e., information binging. The flow theory posits that consumers carrying on information binging would experience flow and keep consuming more. This phenomenon is often being observed in on-demand video streaming and referred to as binge-watching. Binge-watching is defined as watching three or more episodes of a T.V. series in one sitting [7]. A more recent survey reports that more than $80 \%$ of consumers (ages 21-51 years old) view about seven episodes of a T.V. series in a single sitting [4]. Schweidel and Moe [8] show that consumers in the binge-watching state are more likely to continue watching the episodes from the same T.V. series on the Internet channels. However, it is still unclear how the users' content consumption decisions vary in different engagement states.

We intend to answer the following question: How do users' prior spending and network quality impact mobile content consumption decisions? How do the impacts vary in different engagement states? We answer these questions by investigating a large tapstream data set of individual users' reading activities from a mobile app provider in China. In particular, we focus on two content consumption decisions, how many books and how many chapters that a user read on a day. We examine two types of impacting factors: (1) financial factors such as the users' prior spending on the mobile content, and (2) operational factors, such as network delay captured by content loading time.

Exploring the underlying mechanism of individual activities is critical in I.S. and marketing. Since the users' content consumption is driven by their internal engagement states, which evolve over time, we develop a Hidden Markov Model (HMM) to capture these hidden states and the dynamic nature of consumers' choices. Using the large tapstream data set of individual users' reading activities, we aim to identify consumers' latent engagement states and the factors impacting the transition between states and content consumption choices in each state.

The paper is organized as follows. Section 2 reviews the related literature. Section 3 describes the research context and data. The empirical model and preliminary results are presented in Sections 4 and 5 . We conclude the paper by discussing next step in Section 6.

\section{Literature Review}

The study relates to the literature on product trial and sampling. Free trial offers have been widely used by software companies when users are uncertain about the value of the software. The literature has examined various free trial strategies, including the limited time length free trial, the limited version free trial, and hybrid models, and characterized the optimal strategies under different conditions [9-12]. The free trial business models have also been used by Internet platforms selling services such as news, music, and video streaming. Specifically, the platforms offer limited-feature services to users but charge a subscription fee for premium services or offer the full services for a limited trial period. Oestreicher-Singer and Zalmanson [13]) examined a music platform where users can use the basic services for free but have to pay a subscription fee for the premium services. They find a positive relationship between users' subscription decisions and the level of community involvement of users. In particular, a higher level of community engagement is associated with a higher likelihood to subscribe to the premium services and a shorter period of time to consume the free content. Godinho De Matos and Ferreira [14] examine the households' subscription decisions after a free trial period during which the households have free access to an on-demand T.V. service. Surprisingly they found that binge-watchers were less likely to subscribe to the service after the trial period. They attribute the lower subscription rate to the fact that households engaging in binge-watching may have depleted the content libraries and as a result, the value of the service is lower. These studies examine the relationship between users' subscriptions and engagement, whereas our research focuses on a different financial factor, users' prior spendings, and its impact on user engagement.

Download delay is one of the major impediments to growth in online commerce and one of the primary causes of e-commerce failures [15]. The literature has found that online waiting is associated with negative 
business outcomes, such as negative brand attitudes [16], lack of trust [17], interruption of the flow experience [18, 19], problems in maintaining interactivity [20], consumer abandonment [21-23]. How network conditions impact user experience is a popular topic for online games. The literature has mixed results. Some studies show that network QoS is strongly related to player departure and game-playing time [24, 25]. In contrast, others show that download delay does not always bother online consumers or negatively affect their online experiences [26]. This study examines how network delay impacts users' engagement states and mobile content consumption decisions.

Engagement is has been studied in the disciplines of sociology, political science, psychology, and organizational behavior [27-29]. In marketing and service literature, consumer engagement is defined as a psychological state [30-33]) or a psychological process [34]. Following the literature, we consider customer engagement as a transient state occurring within engagement processes developing over time $[35,36]$. There is a growing body of literature studying users' content consumption and engagement online. Kim et al. [37] introduce a concept of mobile users' engagement and use the cognitive-affective-conative state framework to examine users' mobile engagement motivations. Schweidel and Moe [8] examine users' online viewing decisions and responses to the advertisement using a video streaming viewing data set. They find that users are more likely to bingewatching if they watch more episodes from a series in a session. And bingers are less likely to respond to advertisements. Instead of advertising, our study examines ad-free mobile content and users' spending. Huang et al. [38] capture the evolution of online gamers' latent engagement states and state-dependent participation behavior in an online gaming context. Wu et al. [39] develop a framework to capture the underlying mechanism of mobile app usage. Zhang et al. [40] identify user latent engagement states and proposed a personalized targeting strategy based on engagement states. While Huang et al. [38] explore users' motivational factors, Wu et al. [39] examine contextual factors, and Zhang et al. [40] focus on the mobile app's pricing strategies, our study investigates an operational factor, i.e., network delay, in addition to a financial factor, users' prior spending.

\section{Research Context and Data}

We obtained a tap stream data set from a mobile app provider, which is affiliated with one of the largest mobile carriers in China. The app provider maintains a library of comic books. The mobile app is free to install, but the users may have to pay for some comic books to read. Our data contains 1,604,704 reading records on 18,603 new app users for four months from July 2016 to October 2016. All users registered their accounts during this four-month period and had not subscribed to the monthly services. They had to pay for the chapters if the chapters are not free. The app provider normally gives the first few chapters of a book for free and charges a fee ranging from 0.1 RMB to 2 RMB per chapter for the rest of a book. Since mobile app users may read the books anonymously, the app provider uses the unique identifier of mobile devices-International Mobile Equipment Identity (IMEI) number - to identify each user. Each reading record includes a masked IMEI number, user id if the user logged in, the chapter identifier, content loading time, device model, and timestamp. The comic book data set includes the book identifier, book title, chapter identifier, chapter title, the price for each chapter, and book genre. We have also collected the screen size and resolution data for different device models. We aggregate the users' data to the daily level for our analysis. Table 1 shows the variable description.

Table 1. Variable Description

\begin{tabular}{|c|c|}
\hline Variable & Description \\
\hline Breadth $_{i t}$ & $\begin{array}{l}\text { The number of different books that } \\
\text { user } i \text { read on day } t \text {. }\end{array}$ \\
\hline Depth $_{i t}$ & $\begin{array}{l}\text { The number of chapters that user } i \\
\text { read on day } t \text {. }\end{array}$ \\
\hline ViewLength $_{i t}$ & $\begin{array}{l}\text { Total hours that user } i \text { had spent in } \\
\text { reading using the app since the } \\
\text { first visit. }\end{array}$ \\
\hline Tenure $_{i t}$ & $\begin{array}{l}\text { Days elapsed since user } i \text { first } \\
\text { visited the app. }\end{array}$ \\
\hline DaysSince $_{i t}$ & $\begin{array}{l}\text { Days elapsed since user } i \text { 's last } \\
\text { visit. }\end{array}$ \\
\hline LastDelay $_{i t}$ & $\begin{array}{l}\text { Average chapter loading time that } \\
\text { user } i \text { had experienced during the } \\
\text { last visit. }\end{array}$ \\
\hline LastPay $_{i t}$ & $\begin{array}{l}\text { Total content spending (in } \\
\text { Chinese Yuan) that user } i \text { had } \\
\text { incurred during the last visit. }\end{array}$ \\
\hline
\end{tabular}

For this preliminary study, we randomly sample 500 users and retrieve records of the first 30 days of these users, resulting in 14,954 records. Table 2 shows summary statistics of the main variables. Figures 1 and 2 shows the average reading breath and depth across 500 users over the first 30 days.

Table 2. Summary Statistics

\begin{tabular}{lllll}
\hline Variable & Mean & Std & Min & Max \\
\hline
\end{tabular}




\begin{tabular}{lcccc}
\hline Breadth & 0.48 & 1.36 & 0.00 & 32 \\
Depth & 5.23 & 20.85 & 0.00 & 483 \\
ViewLength & 4.08 & 5.08 & 0.01 & 56.08 \\
Tenure & 15.48 & 8.65 & 1 & 30 \\
DaysSince & 6.88 & 7.15 & 0.00 & 29 \\
LastDelay & 8.65 & 7.23 & 0.00 & 99.17 \\
LastPay & 546.61 & 1365.47 & 0.00 & 17570 \\
\hline
\end{tabular}

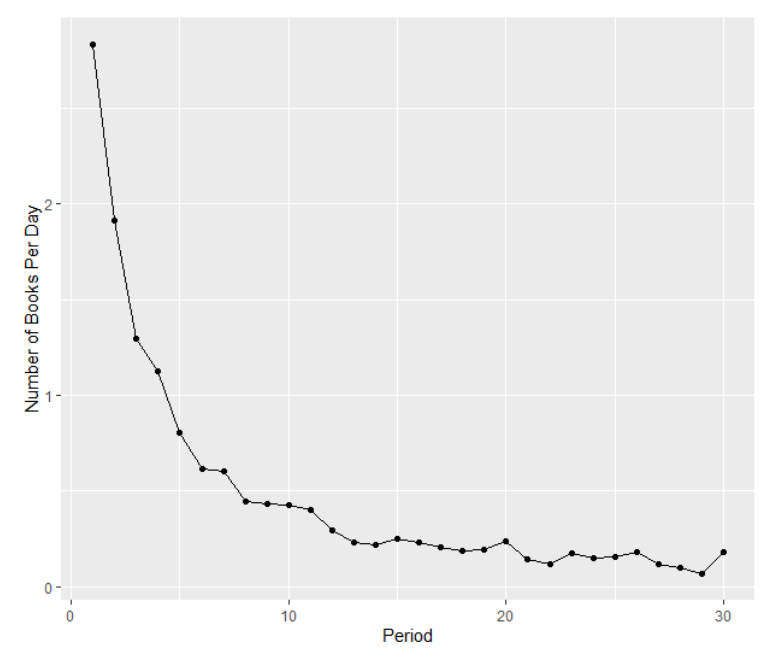

Figure 1. Average Reading Breadth across 500 Users in the First 30 Days

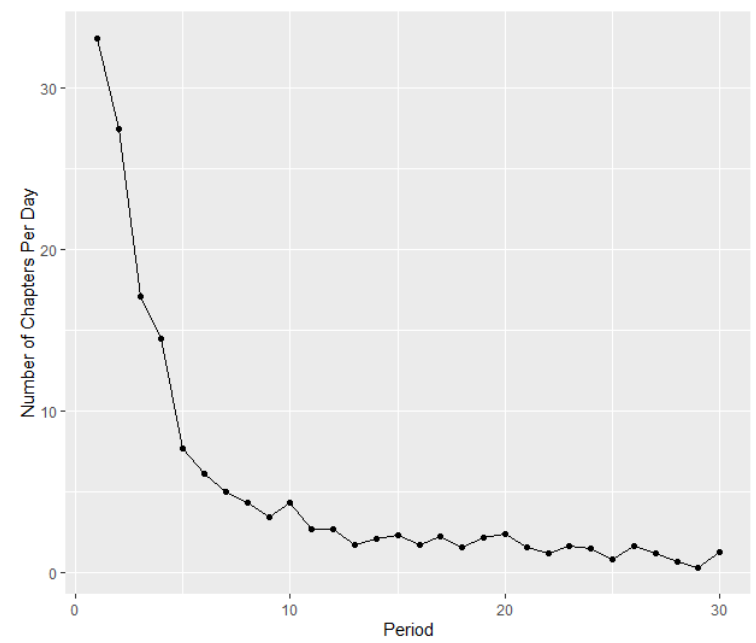

Figure 2. Average Reading Depth across 500 Users in the First 30 Days

\section{Model}

We are interested in examining the numbers of books and chapters that a user chooses to read on a day and the impacting factors. The user choices depend on not only observable time-varying factors but also the users' latent engagement levels. The impact of the observable factors may be state-dependent. We therefore adopt a HMM to model users' latent states which capture the unobserved levels of user engagement with the app (shown in Figure 3).

A standard HMM has three main components:

- The initial state distribution $\boldsymbol{\psi}$, indicating the probability of the user being in state $s$ at the initial time period, $\operatorname{Pr}\left(S_{i, 1}=s_{1}\right), s_{1}=1,2, \ldots, n$;

- A $n \times n$ transition matrix $\boldsymbol{Q}$, explaining the transition probabilities $p\left(S_{i, t}=s_{t} \mid S_{i, t-1}=s_{t-1}\right)$ from $s_{t-1}$ to $s_{t}, s_{t-1}, s_{t}=1,2, \ldots, n$;

- The state-dependent distributions of observed activities: $P\left(Y_{i, t} \mid S_{i, t}=s_{t}\right), s_{t}=1,2, \ldots, n$.

\subsection{Initial State Distribution}

According to the reading patterns in Figures 1 and 2, we assume that users are in the highest state when they start using the app. The probability distribution of initial states is

$$
\boldsymbol{\psi}_{1}=(0,0, \ldots, 1)
$$

\subsection{Transition Matrix}

The transition matrix describes how users' latent states change over time. It is defined as

$$
\boldsymbol{Q}_{i, t-1, t}=\left[\begin{array}{cccc}
p_{i t 11} & p_{i t 12} & \ldots & p_{i t 1 n} \\
p_{i t 21} & p_{i t 22} & \cdots & p_{i t 2 n} \\
& \vdots & \ddots & \vdots \\
p_{i t n 1} & p_{i t n 2} & \cdots & p_{i t n n}
\end{array}\right]
$$

where $0 \leq p_{i t s s^{\prime}} \leq 1, \sum_{s^{\prime}} p_{i t s s^{\prime}}=1, n$ is the total number of states, $p_{i t s s^{\prime}}=\operatorname{Pr}\left(S_{i t}=s^{\prime} \mid S_{i t-1}=s\right)$ is the probability that user $i$ moves from state $s$ in period $t-1$ to state $s^{\prime}$ in period $t$.

More specifically, the nonhomogeneous transition probabilities $p_{i t s s}$ is specified in a multinomial logit form:

$$
\begin{aligned}
& p_{i t s 1}=\frac{1}{1+\sum_{k=2}^{n} \exp \left(\alpha_{s k}+\boldsymbol{X}_{i, t-1} \lambda_{s k}\right)}, \\
& p_{i t s s^{\prime}}=\frac{\exp \left(\alpha_{s s^{\prime}}+\boldsymbol{X}_{i, t-1} \lambda_{s s^{\prime}}\right)}{1+\sum_{k=2}^{n} \exp \left(\alpha_{s k}+\boldsymbol{X}_{i, t-1} \lambda_{s k}\right)^{\prime}}
\end{aligned}
$$

for $s^{\prime} \in\{2,3, \ldots n\}$. Because we normalize the value of transition from any states in period $t-1$ to state 1 in time $t$ as zero, parameters $\lambda_{s s^{\prime}}$, denote the marginal effects of covariates in moving the state to $s^{\prime}$ relative to 1 . The intercept $\alpha_{s s}$ denotes the intrinsic value of 
the transition from state $s$ to $s^{\prime}$ compared with the transition from state $s$ to 1 .

We model a user's propensity to transition as a function of time-varying covariates $\boldsymbol{X}_{\boldsymbol{i}, t-1}$. Since the engagement states capture the users' long-term states, we use the following covariates $\boldsymbol{X}_{i, t-1}$ :

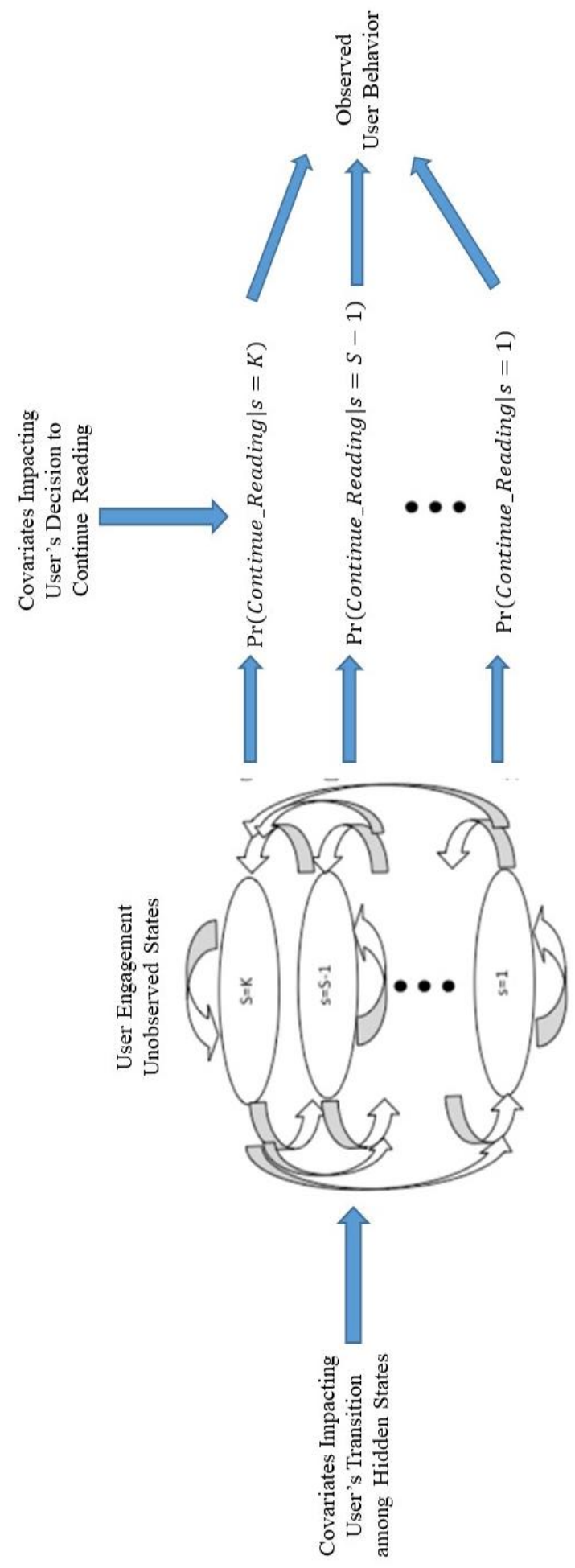

Figure 3. Research Model

- ViewLength ${ }_{i t}$ : Hours that user $i$ spent in reading using the app since the first visit.

- DaysSince $i$ : Days elapsed since user i's last visit. 
- Tenure $_{i t}$ : Days elapsed since user i first visited the app.

\subsection{State-Dependent Reading Outcomes}

We consider two reading outcome variables. Depth $_{i t}$ is the number of chapters that user $i$ read on day $t$. Breath ${ }_{i t}$ is the number of different books that user $i$ read on day $t$. These two variables represent a user's reading decision outcomes given a state.

To control for the potential correlation Breadth $h_{i t}$ and Depth ${ }_{i t}$, we model the reading outcome as a bivariate negative binomial distribution [41]. Let $Y=$ $\left(Y_{1}, Y_{2}\right)$ denote the number of chapters and the number of books that a user reads in a time period. The bivariate negative binomial distribution can be defined as

$$
\begin{aligned}
P\left(Y_{1}=y_{1}, Y_{2}=y_{2}\right)= & \frac{\Gamma\left(y_{1}+y_{2}+\alpha\right)}{y_{1} ! y_{2} ! \Gamma(\alpha)} \\
& \times\left(\frac{\mu_{1}}{\mu_{1}+\mu_{2}+1}\right)^{y_{1}} \\
& \times\left(\frac{\mu_{2}}{\mu_{1}+\mu_{2}+1}\right)^{y_{2}} \\
& \times\left(\frac{1}{\mu_{1}+\mu_{2}+1}\right)^{\alpha}
\end{aligned}
$$

where $\boldsymbol{\alpha}>\mathbf{0}$ and the mean parameters for individual $\boldsymbol{i}$ in state $\boldsymbol{s}$ is

$$
\mu_{i 1,2}^{S}=\exp \left(\boldsymbol{X}_{i, t-1} \boldsymbol{\beta}_{i}^{s}\right)
$$

Since the decision outcomes are driven by short term factors, we use the following time-varying covariates $\boldsymbol{X}_{i, t-1}$ in the decision outcome equation:

- LastDelay ${ }_{i, t-1}$ : Average chapter loading time that user $i$ experienced during the last visit.

- LastPay ${ }_{i, t-1}$ : Total content spending that user $i$ had incurred during the last visit.

\subsection{Likelihood Function}

The likelihood of an observed sequence of reading outcomes is calculated by summing over all possible paths [42]:

$$
\begin{aligned}
L_{i T}\left(Y_{i 1}, Y_{i 2}, \ldots, Y_{i T}\right) & \sum_{s_{1}=1}^{n} \sum_{s_{2}=1}^{n} \ldots \sum_{s_{T}=1}^{n}\left[\operatorname { P r } \left(S_{i 1}\right.\right. \\
= & \left.s_{1}\right) \prod_{\tau=2}^{T} \operatorname{Pr}\left(S_{i \tau}=s_{\tau} \mid S_{i \tau-1}\right. \\
& \left.\left.=s_{\tau-1}\right) \prod_{\tau=1}^{T} \operatorname{Pr}\left(Y_{i \tau} \mid S_{i \tau}=s_{\tau}\right)\right]
\end{aligned}
$$

\section{Preliminary Results and Discussion}

We estimate the models with different numbers of latent states and find that the model with three states fits our data best, i.e., lowest BIC. Table 3 summarizes the characteristics of the three states. In state 1 (low state), users do not read any books at all; in state 2 (medium state), users occasionally read some books; and in state 3 (high state), users are highly engaged and read about 3 books and 16 chapters per day.

Table 4 summarizes the estimation results. When a user is in the low state, she does not read any books or chapters using the app, which suggests the impacts of network delay and expenditure during the last visit are trivial. On the other hand, when a user is in the medium or high state, network delay has a significant negative impact on the user's reading behavior. When a user is in the high state, prior spending during the last visit encourages reading in the current period.

The negative coefficients of Tenure in the transition matrix indicate that the chance of moving to lower states will increase over time. The positive coefficient of ViewLength on the transition to state 3 shows that the more time a user spends in reading using the app, the more engaged she will be in the long term. The days since the last visit, DaySince, may help a user transition to the medium state but not to the high state.

We also calculate the transition matrix given the average value of variables (Table 5). For example, the probability of staying in state 1 is $75.2 \%$, which means the low state is very stable. If a user drops into the low state, she is likely to stay there for many periods. The high state is relatively more sticky than the medium state. A user is likely to stay in state 3 with a probability of $46.3 \%$, which is slightly higher than the $38.1 \%$ probability of staying in state 2 . Furthermore, even if a user is in the medium or high state, she may slide into the low state in the next period with $42.2 \%$ and $41.8 \%$ chances, respectively. 
Table 3. State Description

\begin{tabular}{ccc}
\hline State & Breadth & Depth \\
\hline 1 (Low) & 0 & 0 \\
2 (Medium) & 0.077 & 0.014 \\
3 (High) & 2.908 & 15.585 \\
\hline
\end{tabular}

Table 4. Estimation Results

\begin{tabular}{lll}
\hline \multicolumn{2}{c}{ Variable } & Estimates S.E. \\
\hline State-Dependent Reading Outcome & \\
Breadth intercept state 1 & $-9.493^{*}$ & 0.026 \\
LastDelay state 1 & $2.381^{*}$ & 0.073 \\
LastPay state 1 & $-2.416^{*}$ & 0.074 \\
Depth intercept state 1 & $-10.135^{*}$ & 0.000 \\
LastDelay state 1 & $-0.349^{*}$ & 0.091 \\
LastPay state 1 & $1.266^{*}$ & 0.128 \\
Breadth intercept state 2 & $1.935^{*}$ & 0.010 \\
LastDelay state 2 & $-6.481^{*}$ & 0.327 \\
LastPay state 2 & -0.000 & 0.378 \\
Depth intercept state 2 & $1.768^{*}$ & 0.000 \\
LastDelay state 2 & $-6.926^{*}$ & 0.869 \\
LastPay state 2 & -0.982 & 0.888 \\
Breadth intercept state 3 & $1.291^{*}$ & 0.020 \\
LastDelay state 3 & $-4.390^{*}$ & 0.025 \\
LastPay state 3 & $0.176^{*}$ & 0.013 \\
Depth intercept state 3 & $1.949^{*}$ & 0.000 \\
LastDelay state 3 & $-4.21^{*}$ & 0.000 \\
LastPay state 3 & $0.671^{*}$ & 0.000 \\
Std for Random Parameters & & \\
Breadth intercepts & -0.063 & 0.058 \\
Depth intercepts & $1.174^{*}$ & 0.000 \\
Breadth LastDelay parameter & $-0.055^{*}$ & 0.010 \\
Depth LastDelay parameter & $2.814^{*}$ & 0.000 \\
Transition Matrix & & \\
Intercept state 1 to 2 & $-5.620^{*}$ & 0.510 \\
Intercept state 2 to 2 & -1.092 & 0.844 \\
Intercept state 3 to 2 & $-3.205^{*}$ & 0.408 \\
Intercept state 1 to 3 & $-2.006^{*}$ & 0.127 \\
Intercept state 2 to 3 & $-1.284^{*}$ & 0.320 \\
Intercept state 3 to 3 & -0.047 & 0.167 \\
Viewlength on transition to state 2 & -0.805 & 1.384 \\
DaysSince on transition to state 2 & $3.271^{*}$ & 0.383 \\
Tenure on transition to state 2 & $-4.676^{*}$ & 0.787 \\
Viewlength on transition to state 3 & $0.265^{*}$ & 0.064 \\
DaysSince on transition to state 3 3 & $-1.696^{*}$ & 0.126 \\
Tenure on transition to state 3 & $-0.417^{*}$ & 0.070 \\
Correlation between Breadth and & $0.927^{*}$ & 0.056 \\
Depth & & \\
\hline & & \\
& &
\end{tabular}

Table 5. Transition Matrix

\begin{tabular}{cccc}
\hline State & 1 (Low) & 2 (Medium) & 3 (High) \\
\hline 1 (Low) & 0.752 & 0.035 & 0.213 \\
2 (Medium) & 0.422 & 0.381 & 0.198 \\
3 (High) & 0.418 & 0.119 & 0.463 \\
\hline
\end{tabular}

Our study provides important implications. First, we find that the users may be in three distinct engagement states. In the low state, a user is inactive; in the medium state, a user occasionally browses the library, probably exploring books for reading. In the high state, a user is highly engaged, reading multiple books and chapters. We find that user engagement decreases very quickly. Figure 4 shows how the percentages of users in each state change in the first 30 days. Although initially in the high engagement state, a user is very likely to drop to the medium and low states over time. After about ten days, the majority of users become inactive with no reading activities at all. The inactive users are very likely to stay dormant. But as time goes by, the inactive users may occasionally explore the app and browse books, going up to the medium state. As users spend more time reading, they are more likely to transition to the high state. But still, the medium and high engagement states are very unstable. The dynamics suggest that the low engagement states pose a challenge, and how to activate and engage users is a real problem to app providers.

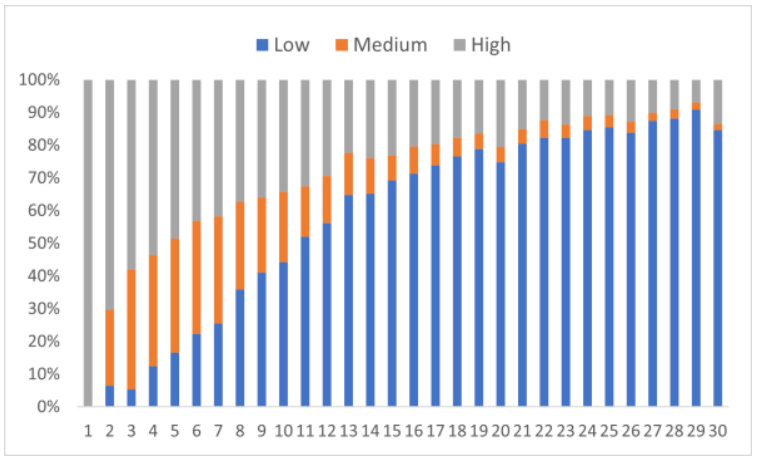

Figure 4. Users' State Distribution in the First
30 Days

Second, network delay discourages users from reading more books and chapters in medium and high engagement states. The negative impact is especially prominent in the high engagement state. The results suggest that high engaged users are likely in the flow state, and slow content loading is more disruptive. Therefore, the app providers should strive to reduce the content loading time, especially for users in the high state. Due to network neutrality, app providers cannot determine or influence how the packets are transmitted over the Internet. However, they can prioritize packet processing on their servers according to network conditions and user engagement. In addition, they may consider preloading some content based on a user's current reading. 
Finally, prior content spending has opposite effects on users' reading choices in the medium and high states. It has a negative or trivial impact in the medium state but a positive impact in the high state. The results suggest that users are less price-sensitive in the high state than in the medium state. The findings of our study provide important implications to app providers on content pricing and user targeting. For example, it is a common practice that app providers publish a couple of chapters/episodes for free for a book and then charge fees for the rest of the chapters. They can monitor the users' daily reading activities and target users based on users' reading engagement states. They can give away free credits or free chapters when users are in the inactive state and the medium state but focus on network delay for users in the high engagement state.

\section{Next Steps}

Next step, we will further improve our models and algorithms. For example, we will empirically estimate the initial state distribution using our data. We will characterize the hidden states, the state transitions, and users' state-dependent content consumption behavior. Finally, based on our calibrated model, we conduct simulations with different content prices and network delays and provide recommendations based on the simulation results.

\section{References}

1. Anderson, M. Mobile Technology and Home Broadband 2019. 2019; Available from: https://www.pewresearch.org/internet/2019/ 06/13/mobile-technology-and-homebroadband-2019/.

2. Lu, K. Growth in mobile news use driven by older adults. 2017; Available from: https://www.pewresearch.org/facttank/2017/06/12/growth-in-mobile-newsuse-driven-by-older-adults/.

3. ComSCORE, The comScore 2017 U.S. Cross-Platform Future in Focus. The comSCORE Whitepaper, 2017.

4. Westcott, K., et al. Digital media trends survey. 2018 [cited 2018 April 23]; Available from: https://www2.deloitte.com/insights/us/en/ind ustry/technology/digital-media-trendsconsumption-habits-survey.html.
5. Watson, L., Humans have shorter attention span than goldfish, thanks to smartphones. The Telegraph, 2015.

6. Adobe. 2019 Adobe Brand Content Survey. 2019; Available from: slideshare.net/adobe/2019-adobe-brandcontent-survey.

7. Spangler, T., Netflix survey: Binge-watching is not weird or unusual. Variety. 2013.

8. Schweidel, D.A. and W.W. Moe, Binge watching and advertising. J. Mark. Journal of Marketing, 2016. 80(5): p. 1-19.

9. Cheng, H.K., S. Li, and Y. Liu, Optimal Software Free Trial Strategy: Limited Version, Time - locked, or Hybrid? Production and Operations Management, 2015. 24(3): p. 504-517.

10. Cheng, H.K. and Y. Liu, Optimal software free trial strategy: The impact of network externalities and consumer uncertainty. Information Systems Research, 2012. 23(2): p. 488-504.

11. Dey, D., A. Lahiri, and D. Liu, Consumer Learning and Time-Locked Trials of Software Products. Journal of Management Information Systems, 2013. 30(2): p. 239268.

12. Lee, Y.-J. and Y. Tan, Effects of different types of free trials and ratings in sampling of consumer software: An empirical study. Journal of Management Information Systems, 2013. 30(3): p. 213-246.

13. Oestreicher-Singer, G. and L. Zalmanson, Content or community? A digital business strategy for content providers in the social age. 2012.

14. Godinho de Matos, M. and P. Ferreira, The Effect of Binge-Watching on the Subscription of Video on Demand: Results from Randomized Experiments. Information Systems Research, 2020. 31(4): p. 1337-1360.

15. Ryan, G. and M. Valverde, Waiting online: A review and research agenda. Internet Research, 2003.

16. Rose, G.M. and D.W. Straub, The effect of download time on consumer attitude toward the e-service retailer. e-Service, 2001. 1(1): p. 55-76.

17. Yoon, S.-J., The antecedents and consequences of trust in online-purchase decisions. Journal of interactive marketing, 2002. 16(2): p. 47-63.

18. Rettie, R., An exploration of flow during Internet use. Internet research, 2001. 
19. Van Beveren, J., R. Widing, and G. Whitwell. Is online flow important to marketers? in Proceedings of the Academy of Marketing Annual Conference, Aston Business School, Birmingham, July. 2003.

20. Stewart, D.W. and P.A. Pavlou, From consumer response to active consumer: Measuring the effectiveness of interactive media. Journal of the Academy of Marketing Science, 2002. 30(4): p. 376-396.

21. Dabholkar, P.A. and X. Sheng, Perceived download waiting in using web sites: a conceptual framework with mediating and moderating effects. Journal of Marketing Theory and Practice, 2008. 16(3): p. 259-270.

22. Kaufman - Scarborough, C. and J.D. Lindquist, $E$ - shopping in a multiple channel environment. Journal of Consumer Marketing, 2002: p. 333-350.

23. Rajamma, R.K., A.K. Paswan, and M.M. Hossain, Why do shoppers abandon shopping cart? Perceived waiting time, risk, and transaction inconvenience. Journal of Product \& Brand Management, 2009.

24. Chen, K.-T., P. Huang, and C.-L. Lei, Effect of network quality on player departure behavior in online games. IEEE Transactions on Parallel and Distributed Systems, 2008. 20(5): p. 593-606.

25. Chen, K.-T., P. Huang, and C.-L. Lei, How sensitive are online gamers to network quality? Communications of the ACM, 2006. 49(11): p. 34-38.

26. Ryan, G., M. del Mar Pàmies, and M. Valverde, $W W W=$ Wait, Wait, Wait: Emotional reactions to waiting on the Internet. Journal of Electronic Commerce Research, 2015. 16(4): p. 261.

27. Achterberg, W., et al., The effect of depression on social engagement in newly admitted Dutch nursing home residents. The Gerontologist, 2003. 43(2): p. 213-218.

28. Resnick, E., Defining engagement. Journal of International Affairs, 2001: p. 551-566.

29. Saks, A.M., Antecedents and consequences of employee engagement. Journal of managerial psychology, 2006.

30. Patterson, P., T. Yu, and K. De Ruyter. Understanding customer engagement in services. in Advancing theory, maintaining relevance, proceedings of ANZMAC 2006 conference, Brisbane. 2006.

31. Vivek, S.D., S.E. Beatty, and R.M. Morgan, Customer engagement: Exploring customer relationships beyond purchase. Journal of marketing theory and practice, 2012. 20(2): p. 122-146.

32. Hollebeek, L.D., Demystifying customer brand engagement: Exploring the loyalty nexus. Journal of marketing management, 2011. 27(7-8): p. 785-807.

33. Mollen, A. and H. Wilson, Engagement, telepresence and interactivity in online consumer experience: Reconciling scholastic and managerial perspectives. Journal of business research, 2010. 63(9-10): p. 919925.

34. Bowden, J.L.-H., The process of customer engagement: A conceptual framework. Journal of marketing theory and practice, 2009. 17(1): p. 63-74.

35. Bryson, C. and L. Hand, The role of engagement in inspiring teaching and learning. Innovations in education and teaching international, 2007. 44(4): p. 349362.

36. Huo, Y.J., K.R. Binning, and L.E. Molina, Testing an integrative model of respect: Implications for social engagement and wellbeing. Personality and Social Psychology Bulletin, 2010. 36(2): p. 200-212.

37. Kim, Y.H., D.J. Kim, and K. Wachter, $A$ study of mobile user engagement (MoEN): Engagement motivations, perceived value, satisfaction, and continued engagement intention. Decision support systems, 2013. 56: p. 361-370.

38. Huang, Y., S. Jasin, and P. Manchanda, "Level Up": Leveraging skill and engagement to maximize player game-play in online video games. Information Systems Research, 2019. 30(3): p. 927-947.

39. Wu, S., et al., How Is Mobile User Behavior Different?-A Hidden Markov Model of Mobile Application Usage Dynamics. A Hidden Markov Model of Mobile Application Usage Dynamics (November 14, 2018), 2018.

40. Zhang, Y., et al., Personalized mobile targeting with user engagement stages: Combining a structural hidden markov model and field experiment. Information Systems Research, 2019. 30(3): p. 787-804.

41. Cameron, A.C. and P.K. Trivedi, Regression analysis of count data. Vol. 53. 2013: Cambridge university press.

42. MacDonald, I.L. and W. Zucchini, Hidden Markov and other models for discrete-valued time series. Vol. 110. 1997: CRC Press. 